\title{
Prensa y poder. Las Provincias, actor político central de la Transición valenciana
}

\author{
Amador IRANZO \\ Universitat Jaume I (Castellón) \\ iranzo@uji.es
}

\begin{abstract}
Resumen:
El diario Las Provincias fue un actor político clave durante la Transición valenciana. Antes de la muerte de Franco, favoreció los movimientos de oposición al franquismo. A partir de 1978, sin embargo, recuperó su tradicional conservadurismo y empezó a utilizar un discurso anticatalanista. Su estrategia común con UCD situó en el centro del debate político los símbolos de identidad de los valencianos, una cuestión que dividió la sociedad y dejó su huella en el Estatuto de Autonomía.
\end{abstract}

Palabras clave: Las Provincias; Transición; UCD; anticatalanismo; prensa

\section{Press and power. Las Provincias, main political actor during the Valencian Transition}

\begin{abstract}
:
Las Provincias daily was a main political actor during the Valencian Transition. Before Franco's death, it supported the opposition movements againts the dictatorship. From 1978, however, the newspaper recovered its traditional conservadurism and started to use an anticatalanism discourse. Its shared strategy with UCD placed the identity simbols of Valencian people on the political arena. This topic divided the people and left its mark in the Statute of Autonomy.
\end{abstract}

Key Words: Las Provincias; Transition; UCD; anticatalanism; press

Referencia normalizada:

Iranzo, A. (2014): Prensa y poder. Las Provincias, actor político central de la Transición valenciana. Historia y Comunicación Social. Vol. 19. Núm. Especial Febrero. Págs. 535-544.

Sumario: 1. Introducción. 2. Metodología. 3. La Primavera de Las Provincias. 4. Cambio de rumbo de Las Provincias. 5. Broseta vincula a Las Provincias con UCD. 6. Conclusiones. 7. Referencias bibliográficas.

\section{Introducción}

La efervescencia política que se vive en España durante los últimos años de la dictadura de Franco encuentra en la prensa —o, al menos, en algunos medios — un válvula de escape que contribuye a configurar lo que se ha dado en llamar como Parlamento de Papel (Cebrián, 1980: 64). Bischoff (1987) niega que se pueda atribuir el título de «motor de cambio» a la prensa en su conjunto. En su opinión, solo merecen ese título, 
en primer lugar, las revistas, entre las que destaca las satíricas, Cuadernos para el Diálogo, Triunfo y Cambio 16. En cuanto a la prensa diaria, el mérito se lo atribuye más bien a determinados periodistas de El Alcázar (1969), Madrid, Informaciones y $Y a$. La prensa también se convierte en esos años en un instrumento para la selección de las élites políticas, además de facilitar la «reconciliación nacional», ya que promueve la colaboración en su seno de personas de ideologías enfrentadas. Aunque la relación de medios que Bischoff sitúa como «motor de cambio» está evidentemente centrada en la prensa madrileña, también en la periferia se pueden encontrar ejemplos de periódicos que preparan la llegada de la democracia. En Valencia, por ejemplo, se puede señalar el caso del diario Las Provincias, tradicional defensor de los intereses de la burguesía, que, a partir de la primavera de 1973, experimenta un periodo de apertura que ha sido etiquetado como La Primavera de Las Provincias. Sin embargo, este periódico no tarda en volver a su tradicional posición conservadora para situarse en el centro del conflicto que, en torno a los símbolos de identidad de los valencianos, se desarrolla durante la Transición (Xambó, 1995, 2001).

Los medios, algunos medios, no solo sirven para alimentar el debate político y dar visibilidad a la oposición durante los últimos años del franquismo, sino que ayudan a configurar la futura democracia (Oneto, 1982: 31). La influencia que tienen Cambio 16 - o Las Provincias, en el caso valenciano-y otros medios de información de esos momentos se ve favorecida por sus conexiones con el poder, entendido en sentido amplio. Así se explica que esos medios reciban un trato más indulgente - en forma de expedientes, sanciones y procedimientos judiciales - que otras voces del panorama periodístico con planteamientos más radicales y autónomos, como es el caso de la revista Triunfo. Como resumió de forma contundente años después Vázquez Montalbán (1995: 175), «Triunfo no ha conseguido tener ningún ministro». Dentro del panorama mediático valenciano, la revista Dos y Dos, aparecida en mayo de 1976, constituye otro buen ejemplo de una publicación con un planteamiento radicalmente progresista y alternativo que sufre el acoso de las autoridades, que la someten a continuas multas, secuestros y procedimientos judiciales. Los casos de Triunfo y Dos y Dos muestran que la prensa auténticamente independiente tiene un influencia muy limitada dentro del Parlamento de Papel de la Transición española porque, como afirma Van Noortwijk (2005), quizá el cuarto poder solo es un poder auténtico cuando mantiene conexiones con el resto de los poderes. De esta forma, la prensa, en términos generales, apoya la reforma política y se desvincula de los planteamientos más radicalmente democráticos de ruptura con el antiguo régimen durante la Transición (Bischoff, 1987; Gómez Mompart, 2009).

\section{Metodología}

Este artículo analiza el papel que jugó el diario Las Provincias durante la Transición valenciana. De acuerdo con Borrat (1989), entendemos que el periódico independiente de información general es un actor político de naturaleza colectiva que 
persigue influir, no conquistar de forma directa el poder institucional. Para ello, actúa de acuerdo con una determinada estrategia y crea alianzas con otros actores para conseguir sus objetivos. El estudio que se realiza de Las Provincias parte, en primer lugar, del análisis de su contenido. De todas formas, este análisis no es suficiente. Si un periódico es un actor político que establece alianzas con otros actores, hay que intentar desentrañar también estas relaciones. Para ello, en la investigación se ha utilizado la técnica cualitativa de la entrevista en profundidad con los protagonistas de la época, ya sean periodistas o políticos. Además del análisis de contenido y la entrevista en profundidad, se han consultado fuentes primarias como el archivo de Emilio Attard, político valenciano y uno de los principales protagonistas de la Transición. El objetivo es, en definitiva, conseguir una aproximación a Las Provincias que supere el estadio de los simples contenidos —el qué- para llegar a desentrañar las razones que se encuentran detrás de estos. El estudio del papel de Las Provincias durante la Transición tiene un interés especial porque, durante ese tiempo, se vivió, especialmente en la ciudad de Valencia y su área metropolitana, un conflicto en torno a los símbolos de identidad de los valencianos que diversos autores coinciden en designar como Batalla de Valencia (Mollà y Mira, 1986; Picó y Reig, 1988; Xambó, 2002; Guia, 2005). En este artículo, sin embargo, se elude el uso de ese término por entender que no refleja de forma adecuada lo ocurrido durante esos años, que cabría definir más bien como una «rebelión, incivil y antidemocrática» (Cucó, 2002: 136).

\section{La primavera de Las Provincias}

Durante los últimos años del franquismo, y bajo la dirección efectiva de la joven María Consuelo Reyna, miembro de la familia editora del periódico, Las Provincias experimenta un continuo proceso de apertura. A pesar de ostentar formalmente el cargo de subdirectora desde 1972, Reyna asumirá de forma progresiva la dirección de facto del diario en detrimento de José Ombuena - veterano periodista que estaba al frente del rotativo desde 1959-, que quedará relegado a un segundo plano: «Ombuena comprendió que su tiempo había acabado y que empezaba el de Consuelo», señala Ricardo Bellveser, redactor en esos momentos de Las Provincias. El punto de inflexión en la línea del periódico lo marca la serie de artículos que Reyna escribe en la primavera de 1973 contra el proyecto de urbanización de El Saler, un paraje natural situado en las afueras de Valencia. A partir de ese momento, el diario, con la entusiasta participación de una redacción mayoritariamente joven y progresista, servirá de apoyo a los movimientos de oposición al franquismo que actuaban en los más diversos frentes: sindical, político, asociaciones de vecinos, etc. Dionisio Vacas, histórico dirigente de Comisiones Obreras, reconocía, años después, el trabajo del diario: «Doy fe personal que fue mucha y casi en solitario la ayuda que nos prestó [al movimiento obrero y vecinal] el diario Las Provincias» (1998: 197). La redacción, y más en concreto el despacho de María Consuelo Reyna, se convierten en punto de encuentro de los principales líderes de todo el espectro de la oposición 
democrática del momento: socialistas, nacionalistas, comunistas, liberales, democristianos, independientes... Vicente Navarro de Luján, dirigente de la UCD-Valencia adscrito al sector democristiano, subraya: «En los años 73, 74 y 75 el único baluarte de oposición era Las Provincias, y por ahí pasábamos todos».

La muerte de Franco aviva el debate en torno a la identidad de los valencianos que, con una perspectiva anticatalanista, había empezado a ser alentado desde unos meses antes por los sectores más próximos al franquismo como aparente estrategia para ganarse el apoyo popular - el anticatalanismo goza de bastante predicamento en ciertos sectores de la sociedad valenciana-y perpetuarse en el poder (Sanz, 1982). El 23 de abril de 1976, Las Provincias responde a este clima con la publicación del editorial «Crisis de identidad», con el que alerta, de forma todavía críptica, del uso torticero que empezaba a hacerse de las cuestiones identitarias. En febrero de 1977, Ramón Pascual Lainosa, teniente de alcalde del Ayuntamiento de Valencia y presidente de la Junta Central Fallera, presenta un ruego en el consistorio en el que denuncia los, a su juicio, intentos de incluir la cultura valenciana dentro de la catalana como paso previo para la creación de los Países Catalanes. El diario replica al texto de Pascual Lainosa con una portada (5 de febrero de 1977) secundada por una columna de Reyna en la que la periodista, después de mostrar su oposición a los Países Catalanes, recuerda que Valencia no será más valenciana por ir contra Cataluña e insta a «actuar por Valencia». La postura del periódico — en este periodo, los editoriales se publican de forma muy esporádica, por lo que la línea editorial de Las Provincias venía marcada por las columnas de María Consuelo Reyna- queda bien definida en estas fechas: rechazo de la propuesta política de los Países Catalanes, denuncia del anticatalanismo como estrategia política y defensa de lo valenciano, entendido en sentido amplio. Es importante subrayar la distinción que Reyna hacía en estos momentos de las vertientes política y cultural de los Países Catalanes: en su columna del 3 de febrero de ese año, la periodista compara los Países Catalanes con Hispanoamérica: en ambas comunidades hay vínculos culturales, especialmente en lo literario, pero no políticos.

Las elecciones del 15 de junio de 1977, ampliamente ganadas por la izquierda en el País Valenciano, y la posterior constitución del Plenario de Parlamentarios abren el camino a la petición de autonomía. El Plenario designa una comisión encargada de organizar los actos festivos de la celebración del 9 de octubre, Día del País Valenciano. Conforme se acerca la fecha señalada, va en aumento el fervor autonomista, del que participa de forma entusiasta Las Provincias con un gran despliegue informativo. Durante los días previos, el diario publica en varias entregas el «Diccionario de la autonomía». En la última, aparecida el mismo 9 de octubre, queda reflejada la postura del diario sobre los símbolos valencianos. Así, en la voz Lengua se afirma que «[1]a lengua hablada mayoritariamente en el País Valenciano es el catalán, en su variante dialectal valenciano»; sobre País Valencià se señala que es la «[d]enominación generalizada modernamente para designar la totalidad de las tierras valencianas»; mientras que en la voz Senyera se admite el uso del término para designar tanto la bandera del País, asociada al pendón real del rey Jaime I de las cuatro barras 
rojas sobre fondo amarillo, como de la ciudad de Valencia, distinguida por su franja vertical azul. La posición reflejada por el diario no puede sorprender: era la aceptada mayoritariamente por la sociedad valenciana. El discurso de Las Provincias durante estos días se puede resumir con una idea: los valencianos deben permanecer unidos para conseguir el autogobierno. El llamamiento a la unidad era una velada respuesta al anticatalanismo que, basado en los símbolos de identidad, amenazaba con fracturar la sociedad valenciana. El anticatalanismo es la principal característica que define el blaverismo, un movimiento de raíz conservadora y españolista, forjado en Valencia en la segunda mitad de la década de los años 70 , que construye una identidad valenciana esencialista definida por su oposición a todo lo catalán (Solves, 2003; Flor, 2011). Reyna reiterará su denuncia de la instrumentalización política del catalanismo, asunto que considera zanjado, en sus columnas de los días 25 de octubre y 31 de diciembre de 1977.

\section{Cambio de rumbo de Las Provincias}

1978 es un año clave en la Transición valenciana porque marca el fin de la etapa aperturista de Las Provincias y el inicio del uso del discurso anticatalanista que había denunciado antes con claridad. En esta evolución tiene una influencia decisiva la estrecha amistad que mantienen María Consuelo Reyna, directora de facto del diario, y Manuel Broseta, abogado y catedrático de Derecho Mercantil, que había jugado un importante papel político en los años previos como presidente de la Junta Democrática del País Valenciano sin adscripción a partido político alguno. Reyna y Broseta se habían conocido cuando Las Provincias iniciaba su Primavera y la periodista le invitó a escribir en el periódico, del que se convirtió en articulista habitual. Según Vicente Navarro de Luján, amigo personal de los dos, «hay una simbiosis total entre ambos personajes», que «van evolucionando juntos». Después de la concesión de la preautonomía al País Valenciano (11 de marzo de 1978), Broseta se convierte en asesor del primer presidente del Consell Preautonòmic [gobierno preautonómico], el socialista Josep Lluís Albiñana, elegido el 10 de abril. Inicialmente, Reyna mantiene su discurso de unidad para conseguir la autonomía, para lo que insiste en la necesidad de que el pueblo no quede dividido por «estériles cuestiones» (columna del 18 de abril). Sin embargo, su posición cambiará radicalmente a raíz de la emisión del programa de televisión «Hora 15» del 19 de mayo, en el que se debatió sobre la idoneidad de usar el adjetivo catalán para referirse al escritor valenciano Ausiàs March. El cambio súbito en la línea de Las Provincias y María Consuelo Reyna resulta todavía más llamativo si se tiene en cuenta que este programa de televisión no era más que una secuela de otro anterior, emitido el 25 de abril, en el que se afirmó que March era un autor que escribía en «lengua catalana» y un «gran poeta catalán medieval». Ni ese programa del 25 de abril ni otros con contenidos similares emitidos anteriormente provocaron reacción alguna por parte de Las Provincias, algo que encaja con la postura defendida hasta entonces por el diario de denunciar el discurso 
anticatalanista. La respuesta al del 19 de mayo es, sin embargo, inmediata. Al día siguiente, Reyna utiliza su columna para denunciar «una maniobra envolvente en torno a Valencia» impulsada por «ciertos sectores pertenecientes a la alta burguesía, cultura y oligarquía catalanas». De esta forma, la periodista avalaba lo que solo unos meses antes había criticado: el uso del fantasma del catalanismo como estrategia política. En días posteriores, Las Provincias dedicará una serie a denunciar una supuesta apropiación por los catalanes del arte, la cerámica o los muebles valencianos. Durante los meses siguientes, y hasta finales de 1978, el diario alternará fases en las que aviva el discurso anticatalanista - especialmente, con la defensa de la senyera con franja azul como símbolo de todo el País Valenciano- con otras de mayor calma.

El cambio en Las Provincias es paralelo al que experimenta por las mismas fechas Manuel Broseta, que se evidencia de forma definitiva con el artículo que el abogado publica en Las Provincias el 23 de julio bajo el título «La paella de "els Països Catalans"». En el texto, reitera los argumentos anticatalanistas utilizados por Las Provincias y María Consuelo Reyna con motivo de la polémica en torno al programa de «Hora 15». El dirigente de UCD Vicente Navarro de Luján, que mantenía una estrecha amistad con el abogado valenciano, admite la «complejidad» del personaje. En su opinión, no hubo tanto una evolución en el pensamiento de Broseta como un cambio de carácter político-estratégico. Albiñana recuerda que, durante esos meses, cuando ocupaba la presidencia del gobierno preautonómico valenciano y Broseta era su asesor, este le ofreció encabezar un nuevo movimiento de carácter regionalista. De acuerdo con su versión — que recoge también Sanz (1982)—, Broseta se había dado cuenta de la orfandad política de la derecha y quería aprovechar el nuevo movimiento blavero que estaba empezando a formarse para situarse como su líder, pero no se atreve a encabezarlo en un primer momento y por eso le hace el ofrecimiento al propio Albiñana con la intención de ocupar su lugar más adelante. Durante un tiempo, Albiñana no da una respuesta definitiva, por lo que Broseta, con el apoyo de Las Provincias, intenta convencerlo. El tratamiento favorable que recibe el presidente del gobierno preautonómico en las páginas del diario (apariciones en portada, artículos de apoyo por parte de Reyna y del propio Broseta) encuentra su momento álgido el 17 de septiembre, cuando el periódico utiliza los datos de una encuesta propia para dedicarle la primera página íntegra con este titular: «Albiñana, considerado por los valencianos como el político más importante del P.V.». Todavía sin respuesta, Broseta realiza un último intento con un artículo que publica en Las Provincias el 12 de noviembre en el que explica su proyecto de una «tercera vía» encabezada por Albiñana. La negativa de este provocará que Las Provincias cambie su línea de forma inmediata y comience a criticar con dureza al presidente.

La evolución que sufren a lo largo de 1978 Las Provincias, de la mano de Reyna, y Broseta es paralela a la que venía experimentando la UCD de la provincia de Valencia bajo la dirección de Emilio Attard. Este, que utiliza el anticatalanismo de forma táctica ya en 1977 para combatir al sector liberal de la coalición y controlar así la formación (Sanz, 1982: 87), se percata también de los réditos electorales que puede reportar el discurso blavero sustentado en los símbolos de identidad de los valencianos. Así, el 
consejo político de UCD-Valencia aprueba, el 27 de febrero de 1978, un texto en el que se defiende el uso por el partido de la senyera con franja azul mientras «el pueblo valenciano no apruebe inequívocamente la bandera que lo represente en todo su territorio». La relevancia del documento reside en que se trata de la primera vez en que UCD-Valencia se pronuncia de forma oficial sobre los símbolos de los valencianos. Ese paso va seguido de otros. Así, Attard y su círculo de colaboradores más cercano, saltándose los órganos de dirección provinciales del partido, llegan, durante 1978, a un acuerdo por escrito con los Grupos de Acción Valencianista (GAV), una entidad blavera que protagonizó varios incidentes violentos durante la Transición. En un informe confidencial que el propio Attard dirige a los «órganos nacionales superiores del partido», el acuerdo con el GAV se justificaba por los «más de 80.000 firmantes» que reunían ${ }^{1}$. De la mano de Attard, por lo tanto, el discurso anticatalanista sustentado sobre los símbolos de identidad se sitúa en el centro de la estrategia de UCD en la provincia de Valencia.

\section{Broseta vinculada a Las Provincias con UCD}

Los caminos paralelos que habían seguido la UCD, por un lado, y Las Provincias / María Consuelo Reyna y Manuel Broseta, por otro, se cruzarán cuando este último decida ingresar en el partido centrista con vistas a las elecciones generales de marzo de 1979. Para Broseta, esa era la única alternativa que tenía de hacer carrera política una vez fracasada la «tercera vía» que había propuesto a Albiñana. En su ingreso jugará un relevante papel la propia Reyna, primero a través de sus columnas (7 y 16 de enero de 1979) y, después, y de acuerdo con el testimonio de Vicente Navarro de Luján, participando con él en las gestiones que acabaron con la entrada del abogado en el partido. El fichaje de Broseta era bien visto por la dirección de UCD en Valencia ya que, de acuerdo con el informe citado anteriormente, serviría para «reforzar considerablemente el apoyo de este periódico [en referencia a Las Provincias]» a la candidatura centrista. Broseta, por lo tanto, vincula a Las Provincias / Reyna con la UCD a partir de enero de 1979. La sintonía entre estos actores será, desde entonces, casi total. Cuando Fernando Abril Martorell, entonces vicepresidente del Gobierno de Adolfo Suárez, llega a Valencia para asumir la dirección del partido antes de las elecciones generales de marzo de 1979, se apoya en Broseta para ejercer su liderazgo. De esta forma, al dúo que formaban María Consuelo Reyna y Manuel Broseta se une Fernando Abril Martorell. «¿Cuál es la diferencia entre María Consuelo, Broseta y Abril? Eran un solo cuerpo. Uno alentaba la ideología del otro y viceversa. Se retroalimentaban», asegura Ricardo Bellveser, periodista de Las Provincias en esos momentos. En agosto de 1982, Attard redacta un informe para el presidente del Gobierno, Leopoldo Calvo Sotelo, en el que dedica un apartado a explicar las relaciones entre Abril Martorell, Broseta y María Consuelo Reyna, y cómo el primero ejerce el control de la UCD de Valencia con la «asistencia» del segundo y la «colaboración» de la periodista ${ }^{2}$. 
La estrecha relación que mantienen a partir de principios de 1979 Las Provincias y UCD es lógica. El discurso simbólico-identitario de raíz anticatalanista es el recurso que utilizan ambos (diario y partido) para buscar la conexión con su público natural: una burguesía desnortada ante el imparable empuje de la izquierda en el País Valenciano. Se trata, no obstante, de un discurso que no permanece invariable, sino que va amoldándose a la evolución de los acontecimientos en función de los intereses de ambos actores. Por ejemplo, a largo de 1978 y 1979 el discurso simbólico se centra en la bandera, especialmente después de que el Consell, con el voto de los partidos de la izquierda, decida, en abril de 1979, adoptar como enseña propia la senyera cuatribarrada con el escudo del País Valenciano, tildada por el blaverismo de «catalanista». Al día siguiente, las páginas de Las Provincias acogen una columna de Reyna y un comunicado de UCD, ambos sumamente críticos, que coinciden en subrayar que la medida fomenta la división de los valencianos y alienta la violencia. Sin embargo, la decisión del nuevo Consell, ya en manos de la formación centrista, de suprimir la bandera del Consell en enero de 1980 dará un giro a la situación. El discurso simbólico pasará, a partir de entonces, a centrarse en la denominación del territorio, una cuestión que apenas había generado polémica hasta ese momento ${ }^{3}$. Tanto Reyna como UCD empezarán a cuestionar con firmeza el término País Valenciano, que antes habían utilizado con normalidad. El discurso identitario anticatalanista tiene, así, un nuevo centro de interés. El ataque definitivo de María Consuelo Reyna llega el 24 de mayo de 1980, cuando la periodista publica su columna «Adiós al país valenciano». El texto coincide con los pronunciamientos de varios comités comarcales UCD en contra de País Valenciano y a favor de usar Reino de Valencia para identificar a todo el territorio valenciano. De esta forma, UCD, con el apoyo de Las Provincias, convertirá la defensa de la denominación Reino de Valencia en el eje central de su estrategia durante la negociación del Estatuto de Autonomía valenciano. Así, el 29 de diciembre de 1981, la Comisión Constitucional del Congreso, con los votos de UCD y de Coalición Democrática, aprueba el proyecto de Estatuto con un cambio en la denominación que debía designar la futura comunidad autónoma: Reino de Valencia sustituye a País Valenciano, término pactado hasta ese momento. Al día siguiente, Las Provincias celebra el cambio a toda portada y Reyna, en su columna, agradece la consecución de Reino a los principales dirigentes de UCD, a Alianza Popular (partido integrado en Coalición Democrática) y al pueblo valenciano. Sin embargo, la intensa campaña que el diario lanza para que la denominación Reino de Valencia supere el siguiente trámite parlamentario fracasa. Ese artículo del Estatuto resulta finalmente rechazado por el pleno del Congreso el 9 de marzo de 1982 y la totalidad del texto es devuelto a la Comisión Constitucional. Las negociaciones posteriores acaban con una solución de compromiso: Comunidad Valenciana será el nombre elegido para designar la futura comunidad autónoma. María Consuelo Reyna, que había defendido la denominación Reino de Valencia hasta la extenuación, acepta el pacto (columna del 22 de abril de 1982) en sintonía con la posición de UCD. Así se cerraba el Estatuto valenciano. 


\section{Conclusiones}

La influencia de Las Provincias y de su directora de facto, María Consuelo Reyna, en la Transición valenciana es indudable. En los años finales de la dictadura, el diario es un referente para los movimientos de oposición al franquismo. Durante esa etapa, se forja la amistad entre Reyna y el abogado Manuel Broseta, clave en los acontecimientos posteriores. Después de la muerte de Franco, Reyna y Las Provincias denuncian el anticatalanismo y secundan el grito casi unánime del pueblo valenciano en defensa de la autonomía. Sin embargo, a partir de 1978 el periódico cambia su discurso y empieza a jugar la baza del anticatalanismo. El ingreso de Broseta en UCD a principios de 1979 establece el vínculo entre el partido y el diario. A partir de ese momento, el trío formado por Reyna, Broseta y Abril Martorell sitúa en el centro de su estrategia, tanto del diario como del partido, el discurso blavero que Emilio Attard había introducido en UCD. Los símbolos de identidad de los valencianos se convierten, desde ese momento, en el centro de la política local. De esta forma, Las Provincias actúa en contra de la unidad de los valencianos que antes había defendido. La sintonía entre el diario y el partido marca un discurso cambiante sobre los símbolos, primero centrado en la bandera y después en la denominación de la futura comunidad autónoma, una discusión que llega a la elaboración del Estatuto de Autonomía y marca la redacción definitiva del texto.

\section{Bibliografía}

ALBIÑANA, Josep Lluís. 11 de marzo de 2010. Entrevista personal.

BELLVESER, Ricardo. 12 de junio de 2009. Entrevista personal

BISCHOFF, H. (1987). «La prensa española en el proceso de transición». En: Cuadernos para el debate, $\mathrm{n}^{\circ} 36$, Madrid: Oficina del Portavoz del Gobierno.

BORRAT, Héctor (1989). El periódico, actor político. Barcelona: Gustavo Gili.

CEBRIÁN, Juan Luis (1980). La prensa y la calle. Madrid: Editorial Nuestra Cultura. CUCÓ, Alfons (2002). Roig i blau. La transició democràtica valenciana. València: Tàndem Edicions.

FLOR, Vicent (2011). Noves glories a Espanya. Anticatalanisme i identitat valenciana. València: Editorial Afers.

GÓMEZ MOMPART, Josep Lluís (2009). «La prensa diaria en el ecosistema comunicativo de la Transición». En QUIROSA-CHEYROUZE Y MUÑOZ, Rafael (ed.) (2009). Prensa y democracia. Los medios de comunicación en la Transición. Madrid: Biblioteca Nueva. p. 103-118.

GUIA I MARÍN, Josep (2005). «La transició política al País Valencià (1974-1977), des d'una perspectiva independentista». En PAGÈS I BLANCH, Pelai (dir.) (2005). La transició democràtica als Països Catalans. Història i memòria. València: Publicacions de la Universitat de València. p. 255-277.

MOLLÀ, Damià; MIRA, Eduard (1986). De impura natione. València: Eliseu Climent. 
NAVARRO DE LUJÁN, Vicente. 15 de junio de 2009. Entrevista personal.

ONETO, José (1982). «La larga marcha de la Transición». En AGUILAR, Miguel Ángel (comp.) (1982). Los medios de comunicación en la frontera democrática. Madrid: UIMP. p. 25-37.

PICÓ, Josep; REIG, Ramir (1988). «Crisis final del franquismo y transición a la democracia». En Cerdà, Manuel (dir.) (1988). Historia del pueblo valenciano. Tomo III. Valencia: Levante. p. 945-964.

SANZ, Jesús, (1982). La cara secreta de la política valenciana. Valencia: Fernando Torres Editor.

SOLVES, Josep (2003). El pensament nacionalista valencià. València: Editorial Denes.

VACAS, Dionisio (1998). «Los sindicatos y su contribución a la recuperación de las libertades». En: GARRIDO MAYOL, Vicente; MARTÍN CUBAS, Joaquín; SOLER SÁNCHEZ, Margarita (coord.) (1998). La transición política en la Comunidad Valenciana. Valencia: Fundación Profesor Manuel Broseta.

VAN NOORTWIJK, Annelies (2005). «La reconstrucción de la razón democrática, o como decir lo indecible (el discurso de Triunfo entre 1962 y 1976)». En VV. AA. (2005). Actes del congrés «La Transició de la dictadura franquista a la democracia». Barcelona: Centre d'Estudis sobre les Ėpoques Franquista i Democràtica - Universitat Autònoma de Barcelona. p. 491-500.

VÁZQUEZ MONTALBÁN, Manuel (1995). «Liberación de añoranzas». En ALTED, Alicia; AUBERT, Paul (ed.) (1995). Triunfo en su época. Madrid: Casa Velázquez / Ediciones Pléyades. p. 171-179.

XAMBÓ, Rafael (1995). Dies de premsa. València: L’Eixam Edicions. (2001). Comunicació, política i sociedat. El cas valencià. València: Edicions $3 i 4$. (2002). «Los medios de comunicación en el País Valenciano». En: Arxius de Ciències Socials, $\mathrm{n}^{\circ}$ 7, Valencia: Facultat de Ciències Socials. p. 223-247.

\section{Notas}

1 Biblioteca Valenciana. Archivo Emilio Attard. Caja 7.

2 Biblioteca Valenciana. Archivo de Emilio Attard. Caja 28.

3 País Valenciano era el término generalmente aceptado para referirse al conjunto del territorio, como demuestra el hecho de que, hasta principios de 1980, el Banco de Valencia o El Corte Inglés usaran esa denominación en su publicidad. 


\section{El autor}

Profesor en el departamento de Ciencias de la Comunicación de la Universitat Jaume I (Castellón) e investigador del Instituto Interuniversitario de Desarrollo Social y Paz (IUDESP), en su sede de Castellón. Doctor en Comunicación por la Universitat de València (2011), licenciado en Comunicación Audiovisual por la misma universidad (2012) y en Ciencias de la Información (sección Periodismo) por el CEU San Pablo - Universidad Politécnica de Valencia (1991). Entre sus líneas de investigación se encuentran las relaciones entre la prensa y el poder, la comunicación de las ONG y la comunicación orientada al cambio social. 